\title{
Using open source accelerometer analysis to assess physical activity and sedentary behaviour in overweight and obese adults
}

\author{
Paul Innerd ${ }^{*}$ D, Rory Harrison and Morc Coulson
}

\begin{abstract}
Background: Physical activity and sedentary behaviour are difficult to assess in overweight and obese adults. However, the use of open-source, raw accelerometer data analysis could overcome this. This study compared raw accelerometer and questionnaire-assessed moderate-to-vigorous physical activity (MVPA), walking and sedentary behaviour in normal, overweight and obese adults, and determined the effect of using different methods to categorise overweight and obesity, namely body mass index (BMI), bioelectrical impedance analysis (BIA) and waistto-hip ratio (WHR).

Methods: One hundred twenty adults, aged 24-60 years, wore a raw, tri-axial accelerometer (Actigraph GT3X+), for 3 days and completed a physical activity questionnaire (IPAQ-S). We used open-source accelerometer analyses to estimate MVPA, walking and sedentary behaviour from a single raw accelerometer signal. Accelerometer and questionnaire-assessed measures were compared in normal, overweight and obese adults categorised using BMI, BIA and WHR.

Results: Relationships between accelerometer and questionnaire-assessed MVPA (Rs $=0.30$ to 0.48 ) and walking (Rs $=0.43$ to 0.58 ) were stronger in normal and overweight groups whilst sedentary behaviour were modest (Rs $=0.22$ to 0.38 ) in normal, overweight and obese groups. The use of WHR resulted in stronger agreement between the questionnaire and accelerometer than BMI and BIA. Finally, accelerometer data showed stronger associations with $\mathrm{BMI}, \mathrm{BIA}$ and WHR (Rs=0.40 to 0.77 ) than questionnaire data ( $\mathrm{Rs}=0.24$ to 0.37 ).

Conclusions: Open-source, raw accelerometer data analysis can be used to estimate MVPA, walking and sedentary behaviour from a single acceleration signal in normal, overweight and obese adults. Our data supports the use of WHR to categorise overweight and obese adults. This evidence helps researchers obtain more accurate measures of physical activity and sedentary behaviour in overweight and obese populations.
\end{abstract}

Keywords: Physical activity, Sedentary behaviour, Measurement, Accelerometer, Questionnaire, Overweight, obese

\section{Background}

Strategies to prevent and treat obesity typically promote increased physical activity [1] and reduced sedentary behaviour [2]. Therefore, accurate measurements of physical activity and sedentary behaviour in overweight and obese individuals are essential. At present, the measurement of physical activity and sedentary behaviour is carried out using either subjective or objective methods [3]. Many studies have used questionnaires to subjectively

\footnotetext{
* Correspondence: paul.innerd@sunderland.ac.uk

School of Nursing and Health Sciences, Faculty of Health Sciences and

Wellbeing, University of Sunderland, Sunderland SR1 3SD, UK
}

assess physical activity and sedentary behaviour $[4,5]$. Questionnaires are inexpensive, easy to administer and allow data to be gathered about physical activity intensity (sedentary, light, moderate, vigorous) and physical activity type, such as sitting and walking, which provides useful contextualisation to the data [6]. However, the subjective nature of questionnaires often results in large measurement error [7].

Accelerometers are becoming increasingly cost-effective and technologically advanced [8]. New accelerometer models such as the ActiGraph GT3X [9], GENEActiv [10], and Axivity AX3 [11] provide access to high-resolution 
$(\leq 100 \mathrm{~Hz})$, raw acceleration data compatible with opensource, freely available analytical methods which estimate physical activity intensity [12], physical activity type [13] and sedentary behaviour [14]. This approach allows the user to obtain a suite of measures from one acceleration signal. Hence, population-based studies such as the National Health and Nutritional Examination Survey (NHANES) [8] the Whitehall II Study [15] and UK Biobank [11] are moving toward the use of raw accelerometer signals. However, the presence of overweight and obesity on the relationship between raw accelerometer and questionnaire-assessed physical activity is poorly understood.

Overweight and obesity is typically classified using different methods across studies, such as body mass index (BMI), bioelectrical impedance analysis (BIA) or waist circumference/waist-to-hip ratio (WHR) [16]. The widespread use of BMI, BIA and WHR is mainly due to their feasibility. However, these methods do not measure the same thing. BMI (normal, overweight, obese) has been criticised for not accurately identifying high adiposity associated with poor health [17], in one study misclassifying $25 \%$ men and $48 \%$ women as obese [18]. The accuracy of BIA (average, high, obese) is heavily influenced by body fat distribution, age and hydration levels varying by up to $10 \%$ [19] and is therefore of limited use in populations other than healthy, euvolemic adults [20]. Some studies report that measures of central adiposity such as WHR (normal, overweight, obese) are better discriminators of unhealthy body composition [21]. However, there is no consensus regarding which method is best.

Raw accelerometer and questionnaire-assessed physical activity is yet to be compared in normal, overweight and obese adults. The aims of this study were to; 1 ) examine whether the relationship between accelerometer and questionnaire-assessed physical activity differs in normal, overweight and obese adults regardless of the method of adiposity grouping; 2) determine whether the method of adiposity grouping (BMI, BIA or WHR) affects the relationship between questionnaire and accelerometerassessed physical activity; 3) quantify the association between accelerometer and questionnaire-assessed physical activity intensity, activity type and sedentary behaviour with adiposity group (BMI, BIA or WHR).

\section{Methods}

\section{Participants}

One hundred twenty adults aged 24 to 60 years were recruited from the University of Sunderland staff and student body via a study advert distributed by email. Selection criteria were age over 18 years, not underweight, no chronic diseases such as cardiovascular disease, stroke, COPD, or reduced functional capacity. Detailed information regarding the purpose and methods used in the study was provided and a medical screening questionnaire was completed. All participants provided written informed consent. All data collection was carried out in accordance with the Declaration of Helsinki. The study protocol was approved by the University of Sunderland Ethics Committee.

\section{Questionnaire-assessed physical activity}

Participants completed a modified version of the International Physical Activity Questionnaire-Short Form (IPAQ-S) at the end of each day. The IPAQ-S is a widely used, 7 item questionnaire in which participants are required to recall their physical activity over the past 7 days. We modified the questionnaire so that participants were asked to recall their physical activity at the end of each day not each week so that questionnaire data was directly comparable to accelerometer data. Questions were as follows: "How much time did you spend doing moderate physical activities like carrying light loads or bicycling at a regular pace?" "How much time did you spend doing vigorous physical activities like heavy lifting, digging, aerobics, or fast bicycling today?" Responses from these questions were combined to give time spent in moderate to vigorous physical activity (MVPA). Further questions were, "How much time did you spend walking today?" and "How much time did you spend sitting today? This may include time spent sitting at a desk, visiting friends, reading, or sitting or lying down to watch television, but not sleeping." Participants also kept a sleep diary, noting the times they went to sleep, when they woke up and if they napped during the day. We then compared questionnaire and accelerometer-assessed measures of MVPA, walking and sedentary behaviour.

\section{Accelerometer-assessed physical activity}

Each participant wore an accelerometer (ActiGraph GT3X+, Actigraph Inc., Pensacola, FL) on their waist for three days. The device was worn continuously to capture continuous raw acceleration data needed for analysis. Three days continuous wear was considered most feasible as participants with high central adiposity can encounter discomfort from the waistband [22]. Accelerometers were initiated to start recording at $0900 \mathrm{~h}$ on day 1 and stop recording $0900 \mathrm{~h}$ on day 4. Data was sampled at $60 \mathrm{~Hz}$, in keeping with other method comparison studies using hip worn raw acceleration data [10] and were stored in gravity $(g)$ units $(1 g=9.81 \mathrm{~m} /$ $\mathrm{s}^{2}$ ). The vector magnitude was taken from the three axes and then subtracted by the value of gravity $(g)$ as in $\sqrt{\mathrm{x} 2+\mathrm{y} 2+\mathrm{z} 2}-1$ after which, negative values were rounded up to zero, referred to as Euclidian Norm Minus One or ENMO [23]. The resulting values are expressed in milligravity $(\mathrm{mg})$, where $1000 \mathrm{mg}=9.81 \mathrm{~m} /$ $\mathrm{s}^{2}$. The average of these values was calculated over a $1 \mathrm{~s}$ 
epoch. Signal processing was done in open-source programme $\mathrm{R}$ (http://cran.r-project.org/). If the reader is interested in replicating these accelerometer analyses with free-living accelerometer data, we recommend using the R-package GGIR which facilitates data cleaning, non-wear detection and the extraction of userdefined acceleration levels. To assess time spent in MVPA we used the cut-point of $70 \mathrm{mg}$ which has accurately classified MVPA from raw acceleration data at the hip [10]. The classification of sedentary and walking activities was carried out using activity type classification which involves the detection of features in the raw acceleration signal to distinguish activities by type [24]. Figure 1 shows visualisation of the raw acceleration signal during selected activities of daily living. Readers interested in reproducing this method are referred to the following study [13]. This analysis detects sitting and supine positions but does not differentiate sedentary behaviour from sleep. Therefore, participants kept a sleep diary to record sleep duration (mins/day), which was subtracted from accelerometer sedentary time before further analysis.

\section{Body composition}

Indicators of overweight and obesity were assessed using BMI, BIA and WHR. BMI was calculated from the participants' weight $(\mathrm{kg})$ divided by height $\left(\mathrm{m}^{2}\right)$. Normal weight was defined as BMI $\leq 24.9$, overweight as BMI
25-29.9 and obese as BMI $\geq 30$ [25]. BIA was used to estimate percent body fat (Helios, Forana, Frankfurt, Germany). The following age-specific cut-offs were used for women; average $22-25 \%$, high $26-36 \%$, obese $>36 \%$, and for men; average 14-20\%, high $21-25 \%$, obese > $25 \%$ based on current standards from the American College of Sports Medicine [26]. Waist and hip circumference was measured with participants standing in a relaxed position using a SECA 201 measuring tape (SECA GmbH\&Co, Hamburg, Germany). WHR ratio was categorised as normal $(<0.8$ for women, $<0.9$ for men), overweight (0.80-0.84 for women, $0.90-0.99$ for men) and obesity ( $>0.85$ for women, $>1.0$ for men) [27].

Demographic factors including sex, smoking status, alcohol intake and educational status were recorded. Alcohol intake was categorised as less than once per week, 1 or 2 times a week and several times a week or daily. Education was assessed by taking the highest qualification attained upon leaving full-time education and was categorised as GCSE/O level, A-level, University degree and Postgraduate.

\section{Statistical analysis}

As the data were not normally distributed, we used nonparametric Spearman's rank correlations with 95\% confidence intervals (CI; derived using Fisher's z transformation) to show associations between sedentary time, walking and MVPA (mins/day) assessed by questionnaire
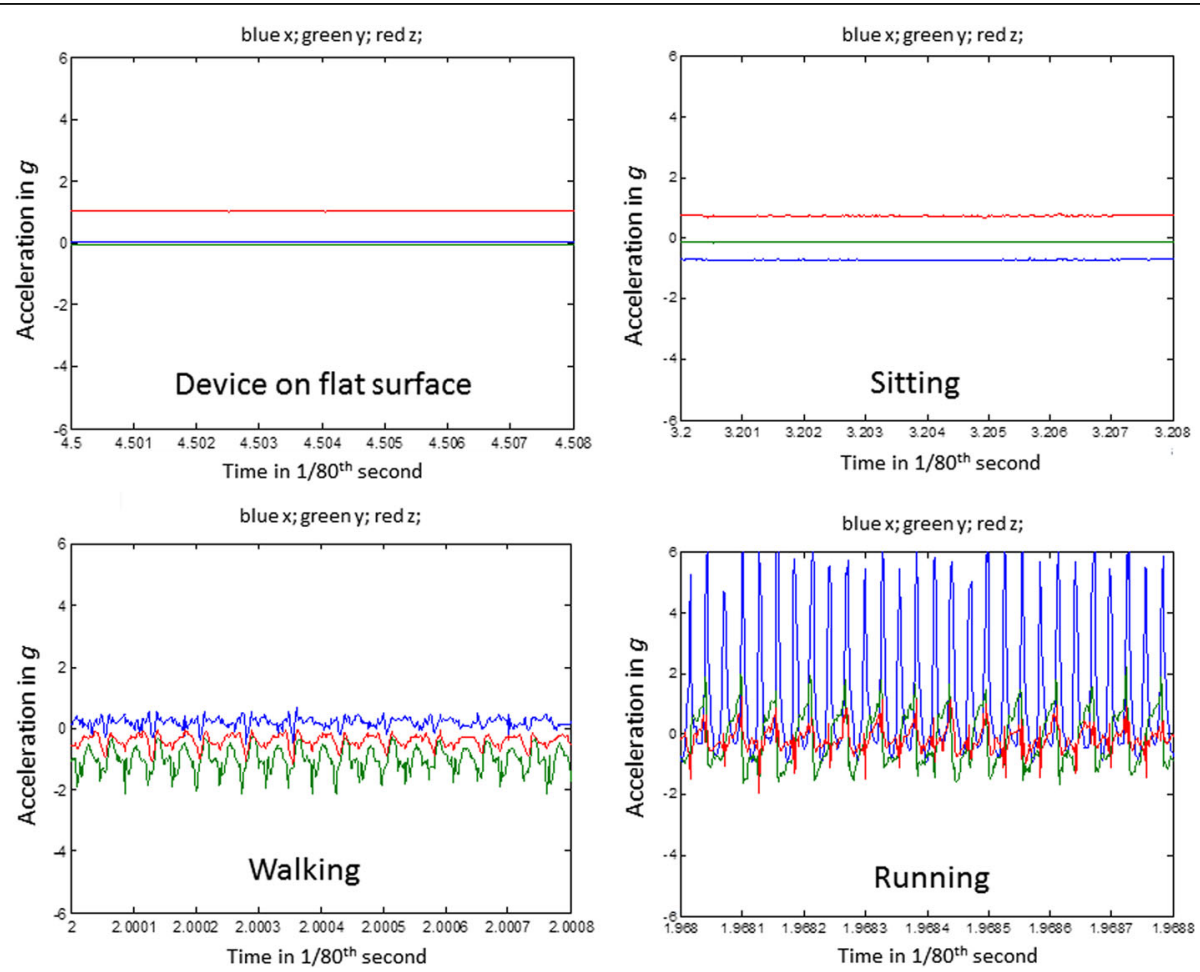

Fig. 1 Raw tri-axial accelerometer output ( $g$ ) showing the device flat on a surface, sitting, walking and running 
and accelerometer. The analyses were stratified by BMI, BIA, WHR, smoking status, alcohol intake and education level to determine whether the relationships between accelerometer and questionnaire-derived measures were stronger in certain adiposity groups and demographic groups. Mean differences between questionnaire and accelerometer-assessed values for sedentary time, walking and MVPA were compared using paired samples t-tests and the bias and variability between the two measurement methods determined using a limits of agreement approach [28]. The associations of adiposity categories with physical activity measures (sedentary time, walking and MVPA) from the questionnaire and accelerometer were examined using Spearman's rank correlations with the adiposity categories as the outcomes. All statistical analyses were performed using SPSS version 21 (SPSS, Inc. Chicago, IL).

\section{Results}

Out of 120 participants, 3 had missing accelerometer data ( $>2 \mathrm{~h}$ of missing data over the three day collection period) and were excluded from the analysis. Therefore, 117 were included in the analytical sample (60 women, 57 men). Table 1 summarises the characteristics of the study population. Participants were aged 24-60 years $(44 \pm 9.2)$. Participants in different adiposity categories were of similar age. For example, normal BMI; $45 \pm$ 8.4 years, overweight BMI; $38 \pm 9.5$ years; obese BMI; 49 \pm 8.7 years. The percentage of smokers $(16 \%)$ was similar to the UK population average (17\%) [29] and participants who reported drinking alcohol weekly (47\%) was

Table 1 Characteristics of the study population

\begin{tabular}{ll}
\hline Characteristics & \\
\hline Sex (\%) & $51(n=60)$ \\
Male & $49(n=57)$ \\
Female & $44 \pm 9$ \\
Age (years) ${ }^{a}$ & $177 \pm 5$ \\
Height (cm) & \\
Weight (kg) $)^{\text {a }}$ & $94 \pm 8$ \\
Smoking status (\%) & \\
Yes & $16(n=19)$ \\
No & $84(n=98)$ \\
Alcohol (\%) & \\
Less than once per week & $53(n=62)$ \\
1 or 2 times a week & $38(n=44)$ \\
Several times a week or daily & $9(n=11)$ \\
Education (\%) & \\
University degree & $79(n=92)$ \\
Postgraduate & $21(n=25)$ \\
\hline
\end{tabular}

${ }^{\mathrm{a}}$ Numbers are in mean \pm SD unless otherwise stated slightly lower than the UK population average (58\%) [30]. The percentage of participants (79\%) who had a degree as their highest qualification was higher than the UK population average (27.2\%) [31].

Table 2 shows the Spearman correlations of accelerometer and questionnaire-assessed sedentary time, walking and MVPA in participants prior to splitting for weight status, stratified by adiposity group (BMI, BIA and WHR) and by sociodemographic group (smoking status, alcohol consumption and education). Modest to high correlations were observed for sedentary time (Rs $=0.49,95 \% \mathrm{CI}: 0.45,0.53)$, walking $(\mathrm{Rs}=0.58,95 \% \mathrm{CI}$ : $0.54,0.61$ ) and MVPA (Rs $=0.56,95 \%$ CI: 0.53, 0.58). For sedentary time, modest correlations were observed across BMI, BIA and WHR adiposity groups ( $\mathrm{Rs}=0.22$, 95\%CI: $0.18,0.25$ to $\mathrm{Rs}=0.38,95 \% \mathrm{CI}: 0.33,0.40)$ and across sociodemographic groups $(\mathrm{Rs}=0.21,95 \% \mathrm{CI}$ : 0.19 , 0.24 to $\mathrm{Rs}=0.32,95 \% \mathrm{CI}: 0.30,0.34)$. Whereas, stronger correlations were observed for walking and MVPA, in participants with normal BMI and normal WHR (Rs = 0.43, 95\%CI: $0.35,0.49$ to $\mathrm{Rs}=0.58,95 \% \mathrm{CI}: 0.42,0.64$ ) overweight BMI and WHR (Rs $=0.30,95 \% \mathrm{CI}: 0.26$, 0.35 to $\mathrm{Rs}=0.48,95 \% \mathrm{CI}: 0.44,0.53)$ but not obese $\mathrm{BMI}$ and WHR (Rs $=0.24,95 \% \mathrm{CI}: 0.18,0.32$ to $\mathrm{Rs}=0.48$, $95 \%$ CI: $0.26,0.42$ ) or any BIA body fat groups (Rs $=0.14$, $95 \%$ CI: $0.08,0.22$ to $\mathrm{Rs}=0.33,95 \% \mathrm{CI}: 0.25,0.39$ ). Modest correlations were also observed across sociodemographic groups for walking and MVPA (Rs $=0.21,95 \% \mathrm{CI}$ : 0.19 , 0.24 to $\mathrm{Rs}=0.36,95 \% \mathrm{CI}$ : $0.33,0.35$ ).

The method of adiposity grouping used (BMI, BIA or WHR) influenced differences between accelerometer and questionnaire-assessed physical activity. Accelerometer and questionnaire-assessed measures were significantly different in participants categorised using BMI and BIA whereas several WHR groups did not reach statistical significance. For example, in obese participants, self-reported sedentary time was underreported by $-195 \mathrm{mins} /$ day $(P<$ $0.001)$ by $-142 \mathrm{mins} /$ day $(\mathrm{P}<0.001)$ and by $-133 \mathrm{mins} /$ day $(P<0.001)$ when classified using BMI, BIA and WHR respectively. Self-reported walking in obese participants was over-reported by $19 \mathrm{mins} /$ day $(P<0.001)$, by $14 \mathrm{mins} /$ day $(\mathrm{P}<0.001)$ and $5 \mathrm{mins} /$ day $(P=0.057)$ when classified using BMI, BIA and WHR respectively. Self-reported MVPA in obese participants was over-reported by $25 \mathrm{mins} /$ day $(\mathrm{P}<0.001)$, by $24 \mathrm{mins} /$ day $(\mathrm{P}<0.001)$ and $12 \mathrm{mins} /$ day $(P=0.052)$ when classified using BMI, BIA and WHR respectively. Similar trends were evident for normal and overweight participants (Table 3).

The associations between adiposity group and accelerometer data were stronger than those between adiposity group and questionnaire data. Table 4 shows Spearman correlations for accelerometer and questionnaireassessed physical activity related to BMI, BIA and WHR adiposity groups. Accelerometer-assessed physical 
Table 2 Spearman correlation (rho) between questionnaire-assessed and accelerometer- assessed sedentary time, walking and MVPA according to adiposity group and sociodemographic characteristics

\begin{tabular}{|c|c|c|c|c|c|c|c|}
\hline & & Seder & & Walki & & MVPA & \\
\hline & & rho & $95 \% \mathrm{Cl}$ & rho & $95 \% \mathrm{Cl}$ & rho & $95 \% \mathrm{Cl}$ \\
\hline All participants & & 0.49 & $0.45,0.53$ & 0.58 & $0.54,061$ & 0.56 & $0.53,0.58$ \\
\hline Adiposity group & & & & & & & \\
\hline BMI category & & & & & & & \\
\hline Normal weight $\leq 24.9$ & $n=37$ & 0.36 & $0.30,0.43$ & 0.46 & $0.40,0.49$ & 0.43 & $0.35,0.49$ \\
\hline Overweight 25-29.9 & $n=37$ & 0.33 & $0.31,0.36$ & 0.33 & $0.29,0.38$ & 0.30 & $0.26,0.35$ \\
\hline Obese $\geq 30$ & $n=43$ & 0.24 & $0.20,0.30$ & 0.34 & $0.26,0.42$ & 0.28 & $0.22,0.36$ \\
\hline BIA body fat $\%$ & & & & & & & \\
\hline Average $14-20$ & $n=43$ & 0.30 & $0.26,0.34$ & 0.31 & $0.27,0.39$ & 0.33 & $0.25,0.39$ \\
\hline High 21-25 & $n=33$ & 0.28 & $0.24,0.31$ & 0.32 & $0.28,0.37$ & 0.21 & $0.17,0.26$ \\
\hline Obese $>25$ & $n=41$ & 0.22 & $0.18,0.25$ & 0.25 & $0.19,0.32$ & 0.14 & $0.08,0.22$ \\
\hline Waist-to-hip ratio & & & & & & & \\
\hline Normal $<0.90$ & $n=43$ & 0.38 & $0.33,0.40$ & 0.58 & $0.42,0.64$ & 0.46 & $0.38,0.52$ \\
\hline Overweight 0.90-0.99 & $n=32$ & 0.34 & $0.32,0.37$ & 0.48 & $0.44,0.53$ & 0.35 & $0.31,0.40$ \\
\hline Obesity > 1.00 & $n=42$ & 0.23 & $0.18,0.25$ & 0.33 & $0.25,0.41$ & 0.24 & $0.18,0.32$ \\
\hline Sociodemographic Measures & & & & & & & \\
\hline Smoking status & & & & & & & \\
\hline Yes & $n=19$ & 0.26 & $0.21,0.29$ & 0.30 & $0.24,0.36$ & 0.28 & $0.20,0.34$ \\
\hline No & $n=98$ & 0.31 & $0.29,0.33$ & 0.34 & $0.28,0.39$ & 0.26 & $0.22,0.31$ \\
\hline Alcohol & & & & & & & \\
\hline Less than once per week & $n=62$ & & & & & & \\
\hline 1 or 2 times a week or less & $n=44$ & 0.29 & $0.23,0.32$ & 0.31 & $0.27,0.35$ & 0.30 & $0.26,0.36$ \\
\hline Several times a week or daily & $n=11$ & 0.30 & $0.29,0.33$ & 0.32 & $0.29,0.35$ & 0.26 & $0.22,0.31$ \\
\hline GCSE/O level & $n=43$ & N/A & & N/A & & N/A & \\
\hline A level & $n=32$ & N/A & & N/A & & N/A & \\
\hline University degree & $n=43$ & 0.32 & $0.30,0.34$ & 0.31 & $0.27,0.35$ & 0.32 & $0.24,0.38$ \\
\hline Postgraduate & $n=32$ & 0.21 & $0.19,0.24$ & 0.36 & $0.33,0.35$ & 0.34 & $0.30,0.39$ \\
\hline
\end{tabular}

Abbreviations: $\mathrm{Cl}$, confidence interval

activity showed modest to strong correlations. For example, correlations according to adiposity group for sedentary time were; 0.49 (BMI), 0.44 (BIA) and 0.53 (WHR), for walking were; 0.58 (BMI), 0.57 (BIA) and 0.70 (WHR), and for MVPA were; 0.54 (BMI), 0.48 (BIA) and 0.67 (WHR). However, questionnaire data showed only modest correlations with adiposity groups (0.24 to 0.37$)$ regardless of which physical activity measure was considered (sedentary behaviour, walking or MVPA).

\section{Discussion}

Technological advances in the objective monitoring of physical activity now make it possible to obtain measures of sedentary behaviour, physical activity intensity and physical activity type from a single, body-worn accelerometer. In this study of normal, overweight and obese adults, we found that; 1) relationships between raw accelerometer and questionnaire-assessed sedentary behaviour were modest across all adiposity groups but walking and MVPA showed stronger associations in normal and overweight groups; 2) the use of WHR instead of BMI and BIA resulted in stronger agreement between accelerometer and questionnaire data; 3 ) associations between adiposity groups and accelerometer data were stronger than associations between adiposity groups and questionnaire data.

This study is the first to obtain several measures of physical activity from raw acceleration data in normal, overweight and obese adults. Methods exist which allow compatibility of raw acceleration signals with output from older devices such as the Actigraph GT1M [32, 33]. Correlations between accelerometer and questionnaireassessed physical activity $($ rho $=0.49$ to rho $=0.58)$ were 


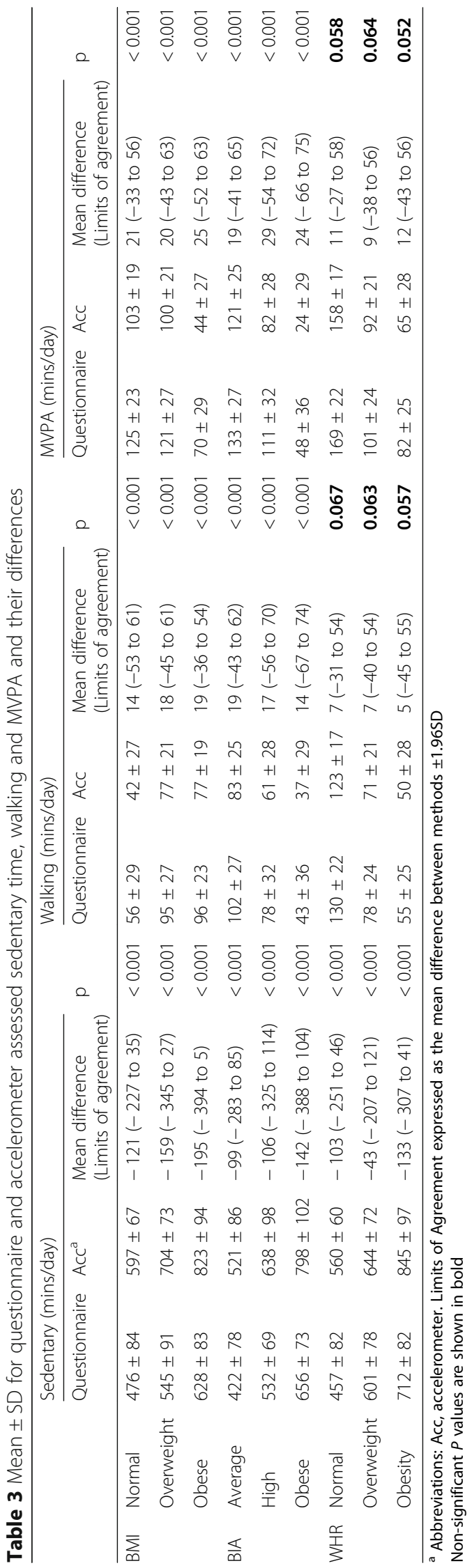


Table 4 Spearman's rank correlations for questionnaire and accelerometer-assessed sedentary time, walking and MVPA with three adiposity categories

\begin{tabular}{lllllll}
\hline & $\begin{array}{l}\text { Sedentary } \\
\text { Questionnaire }\end{array}$ & Acc $^{\mathrm{a}}$ & Walking & & MVPA & \\
& Questionnaire & Acc & Questionnaire & Acc \\
\hline BMl & 0.31 & 0.49 & 0.33 & 0.58 & 0.28 & 0.54 \\
BIA & 0.24 & 0.44 & 0.27 & 0.57 & 0.29 & 0.48 \\
WHR & 0.24 & 0.53 & 0.37 & 0.70 & 0.30 & 0.67 \\
\hline
\end{tabular}

a Abbreviations: Acc, accelerometer

equivalent to the highest reported in similar studies (from rho $=0.09$ to rho $=0.58$ ) using traditional devices [34]. A key recommendation regarding the objective monitoring of physical activity is that data should be collected and saved as raw acceleration signals to allow the storage of large amounts of movement data [35] and facilitate future comparisons of data across studies regardless of which accelerometer is used [36]. However, current recommendations make no reference to the analysis of raw accelerometer data in overweight and obese populations.

Associations between accelerometer and questionnaireassessed sedentary behaviour in the present study were similar to those reported previously $[37,38]$. Sedentary behaviour is an independent risk factor for weight gain [39], meaning research investigating sedentary behaviour in overweight and obese individuals is of increasing importance. Therefore, sedentary behaviour should be explicitly quantified in research and not simply defined by a lack of physical activity [40]. We used a questionnaire which asked specifically about daily sedentary behaviour and an accelerometer analysis which classified sedentary behaviour separately from other activity types [13]. Many accelerometers compress the raw acceleration signal into units called accelerometer counts. Accelerometer counts are generated when acceleration stays above a threshold value for a user defined epoch [41]. This renders some devices unable to differentiate between sedentary behaviours and short-duration, low intensity activities or the removal of the device [42]. Therefore, we opted to identify sedentary behaviour using activity type classification. Activity type classification involves the recognition of signature patterns in the raw acceleration signal which match activity types known by the algorithm [24]. This approach requires large amounts of computing power, hence it has only recently become feasible for use on modern desktop computers in studies involving large numbers of participants [43]. However, the classification analysis we used does not differentiate sleep from sedentary behaviour. Therefore, in the present study, participants kept a sleep diary - noting the times they went to sleep and when they woke up. It is likely the weaker associations observed for sedentary time compared to walking and MVPA is potentially due to the use of self-reported sleep duration. Nevertheless, sleep detection algorithms have recently become available for use with raw acceleration data $[44,45]$ and are incorporated into open-access analytical methods which also monitor MVPA [46]. Therefore, the use of accelerometerbased sleep detection analysis in future work would likely facilitate more accurate measurements of sedentary time. Nevertheless, our activity type analysis has been implemented in sedentary/slow moving populations to identify sedentary time and walking from raw acceleration data to good effect [47].

Walking is the most common type of physical activity, estimated to make up roughly one third of an adult's daily physical activity [48, 49] and is therefore, commonly targeted by weight loss interventions [50-52]. Objective measurement methods such as pedometers [53] and some accelerometers [54] are deemed unsuitable for overweight and obese populations and likely contribute to the weak associations with questionnaireassessed walking reported in previous studies [55-57]. However, as was the case when measuring sedentary behaviour, we used activity classification analysis [13] to detect walking. We found stronger associations between accelerometer and questionnaire-assessed walking in overweight and obese populations than previously reported in the literature [58, 59]. Accelerometer and questionnaire-assessed walking in participants with normal and overweight WHR differed by 7 mins per day and in those with an obese WHR differed by just 5 mins per day. Walking is thought to be detected more accurately in people who are more active [60]. However, obese participants were less active than their leaner counterparts. Therefore the high concordance between accelerometer and questionnaire-assessed walking is likely due to the use of activity classification techniques.

Due to the wealth of evidence associating MVPA with the greatest health benefits, most epidemiological studies assess physical activity expressed in MVPA (mins/day) [61]. We found stronger associations between accelerometer and questionnaire-assessed MVPA than previously reported in the literature. Accelerometer and questionnaire-assessed MVPA from the Whitehall II Study showed modest correlations $(r=0.33)$ [15]. However, authors used wrist-worn accelerometers, which are less burdensome to the participant, but provide a poorer measure of total body movement [42, 62]. Furthermore, care should be taken when monitoring MVPA in overweight and obese populations using accelerometers validated in non-obese adults. Since moderate $(<3-5.99$ METs) and vigorous ( $>6$ METs) physical activity is based on MET cut-points derived from $\mathrm{VO}_{2}$ where $1 \mathrm{MET}=$ $3.5 \mathrm{~mL} / \mathrm{kg} / \mathrm{min}^{-1}$, MVPA will be altered in overweight or obese populations since obesity is associated with reduced cardiorespiratory fitness [63] and diminished metabolic capacity [64]. Unsurprisingly, laboratory and 
free-living experiments suggest that accelerometers detect vigorous activity more accurately than lighter activity [65]. The cut point $70 \mathrm{mg}$, used on this study, has accurately classified MVPA previously [10]. However, this study involved adults with a normal body weight. Therefore, a more modest cut point may be more suitable for overweight and obese adults. Similarly, instead of using the IPAQ-S derived MET values to calculate MVPA [6], self-reported mins/day of moderate and vigorous physical activities were used for analysis. Collectively, these findings go some way in explaining why stronger associations between questionnaire and accelerometer-assessed MVPA were found in overweight and obese adults than previously reported.

Our study compared normal, overweight and obese adiposity groups. We found an overall trend where, irrespective of how adiposity group was classified (BMI, BIA or WHR), lower body weight resulted in stronger relationships between accelerometer and questionnaireassessed sedentary behaviour, walking and MVPA (Rho $=0.14-0.58)$. Similarly, agreement decreased as body weight increased. Previously described studies typically compare accelerometer and questionnaire-assessed physical activity but have not compared normal with overweight/obese groups in the same study. Authors variously recommend questionnaires [66] or advocate the use of accelerometers [67]. This, combined with the use of varying measurement methods, makes direct comparisons with our study difficult. However, it is well established that higher body mass results in increased reporting bias [68]. This could potentially explain the reduction in agreement between accelerometer and questionnaire-assessed physical activity we observed in higher adiposity groups. Nevertheless, care should still be taken when comparing data from studies, where different methods of adiposity classification have been used.

The use of WHR instead of BMI and BIA resulted in stronger agreement between accelerometer and questionnaire-assessed sedentary behaviour, walking and MVPA compared to BMI and BIA. This could be due to the methodological strengths of WHR compared to BMI and BIA. By far the most popular marker of obesity used clinically [69] and in research [70] is BMI. Many studies have also used BIA to provide estimates of fat-mass and fat-free mass [71]. However, BMI is criticised for discounting actual body composition, namely fat-mass and fat-free mass [72] whilst BIA is criticised due to being altered by individual hydration levels [73] and when compared to the "gold standard" dual-energy X-ray absorptiometry, tends to underestimate fat-mass and overestimate fatfree mass [74]. Many studies encourage the use of WHR since it measures central fat unlike BMI and BIA. Studies have shown WHR may be a more informative way of classifying adiposity group (normal, overweight, obese) since this measurement focuses on abdominal or central fat. Excess central fat is linked to increased risk of metabolic syndrome [75], stroke [76], cardiovascular disease [77] and allcause mortality [16]. Despite this, few studies have examined the effect of using different methods of adiposity classification on the relationship between questionnaire and accelerometer-assessed physical activity. We found significant differences between questionnaire and accelerometer-assessed sedentary time, walking and MVPA existed across BMI and BIA adiposity groups but less so for WHR. A previous study which compared physical activity assessed with questionnaire and wrist-worn raw accelerometer data using adiposity groups BMI, waist circumference, fat mass index $(\mathrm{kg} / \mathrm{m} 2)$ and BIA (although BIA was excluded from their analysis as some participants had renal insufficiency and thus, variable hydration levels) reported similar results [78]. Authors reported anthropometric measurements focusing on central adipose measurements were preferable to BMI when assessing physical activity using questionnaire and accelerometer in overweight and obese groups. Combined, these findings indicate WHR differentiated inactivity related excess adiposity most effectively and supports the use of anthropometric measures such as WHR, which estimate central fat, in studies monitoring physical activity and sedentary behaviour in overweight and obese individuals.

The associations between adiposity group and accelerometer data were stronger than those between adiposity group and questionnaire data. Unsurprisingly, these findings correspond with those from several other studies comparing subjective and objective measurement methods $[79,80]$. A recent systematic review comparing accelerometry and questionnaire derived MVPA from both measurement methods advised the use of accelerometer data to obtain most complete physical activity information and reported similarly strong associations with accelerometer derived physical activity and adiposity group compared to questionnaire derived physical activity [81].

Our study is not without limitations. Firstly, although we tried to cover a large socioeconomic range, our participants had a higher than average education level compared to the general UK population. Physical activity in people from higher-socioeconomic groups is more accurately recalled [57, 82]. Secondly, our study does not extend to highest levels of adiposity and does not feature adults classed as extremely obese. Higher adiposity groups are important to reach since they are more difficult to treat [83]. Finally, the use of raw acceleration data in population based studies comes with large data 
processing and data storage needs. Although online platforms which use cloud technology are available more work is needed to make these applications widely accessible and user friendly to facilitate the use of raw acceleration signals in the measurement of physical activity and sedentary behaviour in overweight and obese populations.

\section{Conclusions}

To our knowledge, this is the first study of its kind to compare physical activity and sedentary behaviour derived from raw accelerometer output with those from questionnaire in overweight and obese weight groups. Our findings assist researchers attempting to derive accurate, objective measures of physical activity and sedentary behaviour in overweight and obese adults and recommend the use of WHR. We show how to derive several measures of physical activity, namely sedentary behaviour, walking and MVPA, from a single, raw acceleration signal using open-source data analysis. Future studies can now work toward identifying the aspects of physical activity most important for health in overweight and obese populations by paying closer attention to measurement issues.

\section{Abbreviations}

BIA: Bioelectrical impedance analysis; BMI: Body mass index; Cl: Confidence interval; COPD: Chronic obstructive pulmonary disorder; ENMO: Euclidian Norm Minus One; GGIR: GENActiv and GENEA in R; IPAQ-S: International Physical Activity Questionnaire Short form; MVPA: Moderate-to-vigorous physical activity; NHANES: National Health and Nutritional Examination Survey; Rs: Spearman's rank correlation; WHR: Waist-to-hip ratio

\section{Acknowledgements}

The authors would like to thank the Miss Abbie Taylor, Technician in The Department of Exercise Sciences, University of Sunderland, for her assistance in maintaining the accelerometers, and would also like to thank the participants who kindly volunteered to take part in this study.

\section{Funding}

This study was internally funded by the University of Sunderland.

\section{Availability of data and materials}

The data used in the current study are available from the corresponding author on reasonable request.

\section{Authors' contributions}

$\mathrm{Pl}$ designed the study, $\mathrm{PI}$ and $\mathrm{RH}$ contributed to the acquisition and analysis of the data, $\mathrm{PI}, \mathrm{RH}$ and $\mathrm{MC}$ were involved in the interpretation of the data, $\mathrm{PI}$ drafted the manuscript, $\mathrm{Pl}, \mathrm{RH}$ and $\mathrm{MC}$ were involved in the revision and preparation of the final manuscript. All authors read and approved the final manuscript.

\section{Author's information}

$\mathrm{Pl}$ is a Clinical Exercise Physiologist carrying out research at The University of Sunderland and the NHS. His aims are to improve the measurement of physical activity, sedentary behaviour and sleep across the whole life course in non-clinical and clinical populations to improve our understanding of the prevention and treatment of chronic diseases linked to lifestyle.

\section{Ethics approval and consent to participate}

The study was carried out in accordance with the Helsinki Declaration. The study protocol was approved by the University of Sunderland Research Ethics Committee. All participants gave written informed consent.
Consent for publication

Not applicable.

\section{Competing interests}

None of the authors have competing interests regarding the manuscript.

\section{Publisher's Note}

Springer Nature remains neutral with regard to jurisdictional claims in published maps and institutional affiliations.

Received: 12 June 2017 Accepted: 26 February 2018

Published online: 23 April 2018

\section{References}

1. Maher CA, et al. The independent and combined associations of physical activity and sedentary behavior with obesity in adults: NHANES 2003-06. Obesity (Silver Spring). 2013;

2. Van Dyck D, et al. International study of objectively measured physical activity and sedentary time with body mass index and obesity: IPEN adult study. Int J Obes. 2015;39(2):199-207.

3. Shields M, Connor Gorber S, Tremblay MS. Associations between obesity and morbidity: effects of measurement methods. Obes Rev. 2008;9(5):501-2. author reply 503

4. Golubic R, et al. Rate of weight gain predicts change in physical activity levels: a longitudinal analysis of the EPIC-Norfolk cohort. Int J Obes. 2013; 37(3):404-9.

5. Batsis JA, et al. Normal weight with central obesity, physical activity, and functional decline: data from the osteoarthritis initiative. J Am Geriatr Soc. 2015;63(8):1552-60

6. Lee $\mathrm{PH}$, et al. Validity of the international physical activity questionnaire short form (IPAQ-SF): a systematic review. Int J Behav Nutr Phys Act. 2011:8:115.

7. Lockwood J, et al. Comparison of a physical activity recall questionnaire with accelerometry in children and adolescents with obesity: a pilot study. Pediatr Obes. 2016;

8. Troiano RP, et al. Evolution of accelerometer methods for physical activity research. Br J Sports Med. 2014;48(13):1019-23.

9. Aguilar-Farias N, Brown WJ, Peeters GM. ActiGraph GT3X+ cut-points for identifying sedentary behaviour in older adults in free-living environments. J Sci Med Sport. 2014;17(3):293-9.

10. Hildebrand $M$, et al. Age group comparability of raw accelerometer output from wrist- and hip-worn monitors. Med Sci Sports Exerc. 2014;46(9):1816-24.

11. Doherty A, et al. Large scale population assessment of physical activity using wrist worn accelerometers: the UK biobank study. PLoS One. 2017; 12(2):e0169649.

12. Esliger DW, et al. Validation of the GENEA accelerometer. Med Sci Sports Exerc. 2011:43(6):1085-93.

13. Zhang $\mathrm{S}$, et al. Physical activity classification using the GENEA wrist-worn accelerometer. Med Sci Sports Exerc. 2012:44(4):742-8.

14. Rowlands AV, et al. Sedentary sphere: wrist-worn accelerometer-brand independent posture classification. Med Sci Sports Exerc. 2016:48(4):748-54.

15. Sabia S, et al. Association between questionnaire- and accelerometerassessed physical activity: the role of sociodemographic factors. Am J Epidemiol. 2014;179(6):781-90.

16. Bellocco R, et al. Effects of physical activity, body mass index, waist-to-hip ratio and waist circumference on total mortality risk in the Swedish National March Cohort. Eur J Epidemiol. 2010;25(11):777-88.

17. Shuger $\mathrm{SL}$, et al. Body mass index as a predictor of hypertension incidence among initially healthy normotensive women. Am J Hypertens. 2008;21(6):613-9.

18. Shah NR, Braverman ER. Measuring adiposity in patients: the utility of body mass index (BMI), percent body fat, and leptin. PLoS One. 2012;7(4):e33308.

19. Chouinard LE, et al. Bioelectrical impedance vs. four-compartment model to assess body fat change in overweight adults. Obesity (Silver Spring). 2007; 15(1):85-92.

20. Buchholz AC, Bartok C, Schoeller DA. The validity of bioelectrical impedance models in clinical populations. Nutr Clin Pract. 2004;19(5):433-46.

21. Lee $\mathrm{CM}$, et al. Indices of abdominal obesity are better discriminators of cardiovascular risk factors than BMI: a meta-analysis. J Clin Epidemiol. 2008; 61(7):646-53.

22. Kinnunen $\mathrm{TI}$, et al. Agreement between pedometer and accelerometer in measuring physical activity in overweight and obese pregnant women. BMC Public Health. 2011;11:501 
23. van Hees VT, et al. Separating movement and gravity components in an acceleration signal and implications for the assessment of human daily physical activity. PLoS One. 2013;8(4):e61691.

24. Parkka J, et al. Activity classification using realistic data from wearable sensors. IEEE Trans Inf Technol Biomed. 2006;10(1):119-28.

25. WHO, Obesity: preventing and managing the global epidemic. Report of a WHO consultation. World Health Organ Tech Rep Ser, 2000. 894: p. i-xii, 1-253.

26. Donnelly JE, et al. American college of sports medicine position stand. Appropriate physical activity intervention strategies for weight loss and prevention of weight regain for adults. Med Sci Sports Exerc. 2009;41(2):459-71.

27. Institute, N.I.O.H.A.N.H.L.a.B., Clinical Guidelines on the Identification, Evaluation, and Treatment of Overweight and Obesity in Adults-The Evidence Report. National Institutes of Health. Obes Res, 19986 Suppl 2: p. 51S-209S.

28. Bland JM, Altman DG. Measuring agreement in method comparison studies. Stat Methods Med Res. 1999:8(2):135-60.

29. Allender $\mathrm{S}$, et al. The burden of smoking-related ill health in the UK. Tob Control. 2009;18(4):262-7.

30. Balakrishnan R, et al. The burden of alcohol-related ill health in the United Kingdom. J Public Health (Oxf). 2009;31(3):366-73.

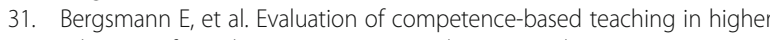
education: from theory to practice. Eval Program Plann. 2015;52:1-9.

32. van Hees, V.T., et al. A method to compare new and traditional accelerometry data in physical activity monitoring. in World of wireless mobile and multimedia networks (WoWMoM), 2010 IEEE international symposium on a. 2010.

33. Rowlands AV, et al. Moving forward with backward compatibility: translating wrist accelerometer data. Med Sci Sports Exerc. 2016;48(11):2142-9.

34. Silsbury Z, et al. Systematic review of the measurement properties of self-report physical activity questionnaires in healthy adult populations. BMJ Open. 2015;15:5

35. Sherar $L B$, et al. International children's accelerometry database (ICAD): design and methods. BMC Public Health. 2011;11:485.

36. Wijndaele $\mathrm{K}$, et al. Utilization and harmonization of adult Accelerometry data: review and expert consensus. Med Sci Sports Exerc. 2015;

37. Clark BK, et al. Past-day recall of sedentary time: validity of a self-reported measure of sedentary time in a university population. J Sci Med Sport. 2016; 19(3):237-41.

38. Gomersall SR, et al. Validity of a self-report recall tool for estimating sedentary behavior in adults. J Phys Act Health. 2015;12(11):1485-91.

39. Clark BK, et al. Relationship of television time with accelerometer-derived sedentary time: NHANES. Med Sci Sports Exerc. 2011;43(5):822-8.

40. Atkin AJ, et al. Methods of measurement in epidemiology: sedentary behaviour. Int J Epidemiol. 2012;41(5):1460-71.

41. Rowlands AV, Stiles VH. Accelerometer counts and raw acceleration output in relation to mechanical loading. J Biomech. 2012;45(3):448-54.

42. Rosenberger ME, et al. Estimating activity and sedentary behavior from an accelerometer on the hip or wrist. Med Sci Sports Exerc. 2013;45(5):964-75

43. Hiden $\mathrm{H}$, et al. Developing cloud applications using the e-Science Central platform. Philos Trans A Math Phys Eng Sci. 2013;371(1983):20120085.

44. van Hees VT, et al. A novel, open access method to assess sleep duration using a wrist-worn accelerometer. PLoS One. 2015;10(11):e0142533.

45. Wrzus C, et al. A new approach for assessing sleep duration and postures from ambulatory accelerometry. PLoS One. 2012;7(10):e48089.

46. Rowlands AV, et al. Raw accelerometer data analysis with GGIR R-package: does accelerometer brand matter? Med Sci Sports Exerc. 2016;

47. Innerd $\mathrm{P}$, et al. A comparison of subjective and objective measures of physical activity from the Newcastle 85+ study. Age Ageing. 2015;44(4):691-4.

48. Valenti G, Bonomi AG, Westerterp KR. Walking as a contributor to physical activity in healthy older adults: 2 week longitudinal study using Accelerometry and the doubly labeled water method. JMIR Mhealth Uhealth. 2016;4(2):e56

49. Audrey S, Procter S, Cooper AR. The contribution of walking to work to adult physical activity levels: a cross sectional study. Int J Behav Nutr Phys Act. 2014;11(1):37

50. Levine JA, et al. The role of free-living daily walking in human weight gain and obesity. Diabetes. 2008;57(3):548-54.

51. Jiang $\mathrm{H}$, et al. Can physical activity reduce excessive gestational weight gain? Findings from a Chinese urban pregnant women cohort study. Int J Behav Nutr Phys Act. 2012;9:12.

52. Bonomi AG, et al. Weight-loss induced changes in physical activity and activity energy expenditure in overweight and obese subjects before and after energy restriction. PLoS One. 2013;8(3):e59641.
53. Saunders TJ, et al. Validity of SC-StepRx pedometer-derived moderate and vigorous physical activity during treadmill walking and running in a heterogeneous sample of children and youth. BMC Public Health. 2014;14:519.

54. Papadopoulos A, et al. Differentiating walking from other activities of daily living in older adults using wrist-based accelerometers. Curr Aging Sci. 2015; 8(3):266-75.

55. Barnett A, et al. Validity of treadmill- and track-based individual calibration methods for estimating free-living walking speed and VO2 using the Actigraph accelerometer. BMC Sports Sci Med Rehabil. 2015;7:29.

56. Barnett $A$, et al. Measuring moderate-intensity walking in older adults using the ActiGraph accelerometer. BMC Geriatr. 2016;16(1):211.

57. Conklin Al, et al. Socioeconomic status, financial hardship and measured obesity in older adults: a cross-sectional study of the EPIC-Norfolk cohort. BMC Public Health. 2013;13:1039.

58. Fitzgerald $\mathrm{L}$, et al. Accelerometer validation of questionnaires used in clinical settings to assess MVPA. Med Sci Sports Exerc. 2015;47(7):1538-42.

59. Oyeyemi AL, et al. Accelerometer-determined physical activity and its comparison with the international physical activity questionnaire in a sample of Nigerian adults. PLoS One. 2014;9(1):e87233.

60. Ainsworth $\mathrm{BE}$, et al. Recommendations to improve the accuracy of estimates of physical activity derived from self report. J Phys Act Health, 20129 Suppl. 1:S76-84.

61. Stabler ME, Giacobbi PR Jr, Fekedulegn DB. Association of television viewing time with overweight/obesity independent of meeting physical activity guidelines: do joint exposures yield independence? J Epidemiol. 2013;23(5):396-7.

62. Swartz AM, et al. Estimation of energy expenditure using CSA accelerometers at hip and wrist sites. Med Sci Sports Exerc. 2000;32(9 Suppl):S450-6.

63. Raiber $L$, et al. Accelerometer thresholds: accounting for body mass reduces discrepancies between measures of physical activity for individuals with overweight and obesity. Appl Physiol Nutr Metab. 2017:42(1):53-8.

64. Sergi G, et al. Resting VO2, maximal VO2 and metabolic equivalents in freeliving healthy elderly women. Clin Nutr. 2010;29(1):84-8.

65. Welch WA, et al. Classification accuracy of the wrist-worn gravity estimator of normal everyday activity accelerometer. Med Sci Sports Exerc. 2013; 45(10):2012-9.

66. Regaieg $\mathrm{S}$, et al. The reliability and concurrent validity of a modified version of the international physical activity questionnaire for adolescents (IPAQ-A) in Tunisian overweight and obese youths. Med Princ Pract. 2016;25(3):227-32.

67. Bell $R$, et al. Measuring physical activity in pregnancy: a comparison of accelerometry and self-completion questionnaires in overweight and obese women. Eur J Obstet Gynecol Reprod Biol. 2013;170(1):90-5.

68. Connor Gorber, S. and M.S. Tremblay, The bias in self-reported obesity from 1976 to 2005: a Canada-US comparison. Obesity (Silver Spring), 2010 18(2): p. 354-61.

69. $\mathrm{Ng} \mathrm{M}$, et al. Global, regional, and national prevalence of overweight and obesity in children and adults during 1980-2013: a systematic analysis for the global burden of disease study 2013. Lancet. 2014;

70. Holme I, Tonstad S. Survival in elderly men in relation to midlife and current BMI. Age Ageing. 2015;44(3):434-9.

71. Kivimaki $\mathrm{M}$, et al. Optimal form of operationalizing $\mathrm{BMI}$ in relation to allcause and cause-specific mortality: the original Whitehall study. Obesity (Silver Spring). 2008;16(8):1926-32.

72. Kim KS, et al. A comparison between BMI and Conicity index on predicting coronary heart disease. the Framingham Heart Study Ann Epidemiol. 2000; 10(7):424-31.

73. Elia M. Body composition by whole-body bioelectrical impedance and prediction of clinically relevant outcomes: overvalued or underused? Eur J Clin Nutr. 2013;67(Suppl 1):S60-70.

74. Gaba A, et al. Comparison of multi- and single-frequency bioelectrical impedance analysis with dual-energy X-ray absorptiometry for assessment of body composition in post-menopausal women: effects of body mass index and accelerometer-determined physical activity. J Hum Nutr Diet. 2015:28(4):390-400.

75. Kim JY, et al. Comparability and utility of body composition measurement vs. anthropometric measurement for assessing obesity related health risks in Korean men. Int J Clin Pract. 2013;67(1):73-80.

76. Abete l, et al. Association between different obesity measures and the risk of stroke in the EPIC Spanish cohort. Eur J Nutr. 2015;54(3):365-75. 
77. Gateva AT, Kamenov ZA. Markers of visceral obesity and cardiovascular risk in patients with polycystic ovarian syndrome. Eur J Obstet Gynecol Reprod Biol. 2012;164(2):161-6.

78. Sabia S, et al. Physical activity and adiposity markers at older ages: accelerometer vs questionnaire data. J Am Med Dir Assoc. 2015;16(5):438.e7-13.

79. Foong $Y C$, et al. The association between physical activity and reduced body fat lessens with age - results from a cross-sectional study in community-dwelling older adults. Exp Gerontol. 2014;55:107-12.

80. Harris TJ, et al. A comparison of questionnaire, accelerometer, and pedometer: measures in older people. Med Sci Sports Exerc. 2009;41(7): 1392-402.

81. Skender $\mathrm{S}$, et al. Accelerometry and physical activity questionnaires - a systematic review. BMC Public Health. 2016;16:515.

82. Bauman AE, et al. Correlates of physical activity: why are some people physically active and others not? Lancet. 2012:380(9838):258-71.

83. Flum DR, et al. Perioperative safety in the longitudinal assessment of bariatric surgery. N Engl J Med. 2009;361(5):445-54.

Submit your next manuscript to BioMed Central and we will help you at every step:

- We accept pre-submission inquiries

- Our selector tool helps you to find the most relevant journal

- We provide round the clock customer support

- Convenient online submission

- Thorough peer review

- Inclusion in PubMed and all major indexing services

- Maximum visibility for your research

Submit your manuscript at www.biomedcentral.com/submit 